\title{
Extracción de ADN bacteriano a partir de cuerpos de agua de uso agrícola
}

\section{Extraction of Bacterial DNA from Water Sources of Agricultural Use}

\author{
Nicolas Forero-Pineda' ${ }^{1}$ \\ Johana Marin-Suarez ${ }^{2}$ \\ Fabio E. Forero-Ulloa ${ }^{3}$ \\ Andrés Gómez-Palacio ${ }^{4}$
}

DOI: https://doi.org/10.19053/01228420.v18.n1.2021.11703

RESUMEN: Dentro del proceso agrícola se usan diferentes tipos de cuerpos de agua, por esto el conocimiento de la productividad del cultivo debe incluir el entendimiento del flujo del material microbiológico presente en las fuentes de agua usadas. La última aproximación metodológica pretende la identificación de comunidades bacterianas mediante el análisis de secuencias de ADN reveladas en muestras ambientales. Debido a la alta contaminación que pueden tener las muestras ambientales es importante llevar a cabo un proceso de extracción de ADN óptimo que permita realizar un posterior análisis molecular por métodos basados en PCR. Por esta razón, el objetivo del presente artículo es describir un protocolo químico de extracción de ADN bacteriano para cuerpos de agua usados en la actividad agrícola local, que sea sencillo, eficiente y rápido de aplicar, para obtener ADN de calidad. A partir de muestras de agua obtenidas del lago de Tota (Boyacá, Colombia), se extrajo ADN bacteriano mediante un protocolo químico modificado y comparado con un método estándar comercial. Como resultado se obtuvo ADN bacteriano con una concentración superior a $140 \mathrm{ng} / \mathrm{ul}$ y una pureza $>1,7 \mathrm{~A} 260 / 280$, resultados similares a los obtenidos con el método estándar comercial con una concentración máxima de 45,94 ng/ul y una pureza superior a 1,8A260/280. Los resultados sugieren que este protocolo de extracción de ADN es un método rápido y de bajo costo con el cual se obtiene ADN de elevada calidad y pureza que puede ser utilizado en cualquier análisis molecular.

PALABRAS CLAVE: microbiológico, contaminación, ADN bacteriano, agrícola.
ABSTRACT: Within the agricultural process different types of water bodies are used, therefore knowledge of crop productivity must include the understanding of the flow of microbiological material present in the used water sources. The last methodological approach aims at the identification of bacterial communities through the analysis of DNA sequences existent in environmental samples. Due to the high contamination that environmental samples may have, it is important to perform an optimal DNA extraction process that allows subsequent molecular analysis by PCR-based methods. For this reason, the objective of this article is to describe a chemical protocol for the extraction of bacterial DNA from water bodies used in local agricultural activities that is simple, efficient, and quick to apply to get good-quality DNA. Bacterial DNA was extracted from water samples obtained from Tota Lake (Boyacá, Colombia) using a modified chemical protocol and compared with a standard commercial method. As a result, bacterial DNA was obtained with a concentration higher than $140 \mathrm{ng} / \mathrm{ul}$ and a purity $>1.7 \mathrm{~A} 260 / 280$, results which are similar to those obtained with the commercial standard method with a maximum concentration of $45.94 \mathrm{ng} / \mathrm{ul}$ and a purity higher than 1.8 A260/280. The results suggest that this DNA extraction protocol is a fast and low-cost method with which high quality and purity DNA is obtained that can be used in any molecular analysis.

KEYWORDS: microbiological, contamination, bacterial DNA, agricultural.

1 Biólogo. Maestría en Ciencias Biológicas. Universidad Pedagógica y Tecnológica de Colombia, Escuela de Ciencias Biológicas. Grupo de investigación en Ciencias Biomédicas (GICBUPTC), Tunja 150003, Colombia.nicolas.forero@uptc.edu.co (D) https://orcid.org/0000-0003-2421-5674.

2 Biólogo. Maestría en Ciencias Biológicas. Universidad Pedagógica y Tecnológica de Colombia, Escuela de Ciencias Biológicas. Grupo de investigación en Ciencias Biomédicas (GICBUPTC), Tunja 150003, Colombia. johana.marin01@uptc.edu.co (D) https://orcid.org/0000-0001-6988-8093.

3 M.Sc. Universidad Pedagógica y Tecnológica de Colombia, Facultad en Ciencias Agropecuarias, Grupo de Investigaciones CIPSO. Tunja, Boyacá, Colombia. fabio.forero@uptc.edu.co (D) https://orcid.org/0000-0001-6815-6579.

4 Ph.D. Universidad Pedagógica y Tecnológica de Colombia. Escuela de Ciencias Biológicas. Laboratorio de Investigación en Genética Evolutiva. Universidad Pedagógica y Tecnológica de Colombia, Tunja, Boyacá, Colombia. andres.gomez04@uptc.edu.co (D) https://orcid.org/0000-0002-1069-9199. 


\section{Introducción}

Las comunidades microbianas aportan una gran cantidad de servicios ecosistémicos a las fuentes hídricas; por ejemplo, actúan en la descomposición de contaminantes y biorremediación (Fennell et al., 2011; Reed \& Martiny, 2013), y son usadas como herramientas de biomonitoreo de calidad del agua (Pawlowski et al., 2018). Adicionalmente, el conocimiento de la composición de las comunidades microbianas permite entender diversos aspectos de la regulación de los ciclos biogeoquímicos (Causarano et al., 2007), los efectos en la salud humana y animal, y la productividad de los cultivos (Schmidt et al., 2019), además, dependiendo del potencial funcional de la comunidad, puede afectar o modelar el hábitat para ocupar nichos particulares (Mouillot et al., 2013; Huang et al., 2014). Es por eso que el conocimiento de la salud y la productividad de un cultivo agrícola se puede basar en el entendimiento del flujo del material y energía dentro de las comunidades microbianas presentes en el suelo, como en el agua usada para tal fin (Röling et al., 2007).

Para el caso de las bacterias es bien conocido que estas son las responsables de los ciclos de carbono, nitrógeno, fósforo y azufre, los cuales son esenciales en el mantenimiento de la dinámica ecológica de los ecosistemas (Fuhrman, 2009; Peter \& Sommaruga, 2016). Por tal razón, se considera que determinar los patrones de diversidad de microorganismos, así como establecer las interacciones entre taxones y los roles funcionales asociados a características fisicoquímicas de los cultivos, son aspectos fundamentales para entender el funcionamiento de los nichos ecológicos ocupados por las comunidades bacterianas y su impacto en la dinámica del ecosistema (Chaffron et al., 2010).

El estudio de la composición y la actividad funcional de las comunidades bacterianas en los diferentes ecosistemas es un tema de creciente interés en ámbitos multidisciplinarios, pues esta información es útil para comprender el estado y la dinámica de los sistemas de cultivos. La más reciente aproximación metodológica en estos estudios pretende la identificación de las comunidades bacterianas mediante el análisis de secuencias de ADN presentes en muestras ambientales (Chaudhary et al., 2018). El surgimiento de herramientas de secuenciación masiva y los análisis metagenómicos en muestras ambientales se conocen como ecogenómica o bioinformática ambiental (Pawlowski et al., 2018). Esta aproximación permite evaluar no solo la composición taxonómica presente en las muestras, sino también estimar parámetros de diversidad bacteriana y su relación con factores fisicoquímicos en procesos funcionales de los ecosistemas (Boon et al., 2014; George et al., 2010; Gilbert et al., 2014; Gilbert et al., 2010; Payne et al., 2017; Venter et al., 2004).

Con el fin de realizar análisis metagenómicos exitosos, se requiere un protocolo eficiente para la extracción de ADN total de una comunidad que está presente en una muestra (Hu et al., 2010). Por esta razón, se considera que 
un protocolo de extracción de ADN genómico es esencial para un posterior análisis confiable (Bag et al., 2016). Por consiguiente, la necesidad de un aislamiento y extracción de ADN genómico de alta calidad se considera como un paso limitante para la construcción de bibliotecas metagenómicas y el análisis de toda la diversidad microbiana presente en la muestra original (Kathiravan et al., 2015). En este sentido, diversos estudios han desarrollado e informado la implementación de protocolos químicos de extracción de ADN genómico para diferentes tipos de muestras (i. e., suelo y agua) (Hassan et al., 2018; Sagar et al, 2014; Zelaya-Molina et al., 2011). Sin embargo, se continúa buscando optimizar aún más el proceso de extracción para asegurar una buena concentración y pureza (relación proteínas-ácidos nucleicos) del ADN extraído, el cual es la base primordial para el proceso de secuenciación y análisis bioinformático.

A pesar de la importancia del uso de protocolos para una buena extracción de ADN para estudios metagenómicos, en Colombia se han realizado pocos estudios sobre muestras de origen ambiental (i. e., suelo y agua) (Bohorquez et al., 2012; Delgado-Serrano et al., 2014; Jiménez et al., 2012; Jiménez et al., 2012). Por esta razón, el éxito de los estudios en aproximaciones metagenómicas de muestras ambientales se basa principalmente en la obtención de ADN metagenómico de alta calidad, y para esto es indispensable poseer un protocolo de fácil acceso, bajos costos y con resultados parecidos a los de los kits comerciales. Por eso, el objetivo del presente trabajo es la modificación de un protocolo de extracción rápida, que permita de forma eficiente y reproducible obtener ADN metagenómico en muestras de agua de uso agrícola del lago de Tota, (departamento de Boyacá, Colombia).

\section{Materiales y Métodos}

\section{A. Área de estudio}

Las muestras fueron obtenidas del lago de Tota, el cual está ubicado a 3015 m s.n.m., es el lago de alta montaña más grande de Colombia (cerca de 60 $\mathrm{km}^{2}$ de extensión) y está rodeado de cultivos como papa y cebolla de rama. Este lago es la fuente principal de agua para los municipios de la cuenca y es utilizado tanto para el consumo humano como para el riego los cultivos.

\section{B. Obtención de la muestra y filtración}

El muestreo se realizó en diciembre de 2018. La muestra de agua se obtuvo del centro del lago al nivel de la zona afótica, que se caracteriza por presentar condiciones idóneas para una alta presencia de bacterias (i. e., bajo contenido de oxígeno y bajas temperaturas). La muestra inicial fue de $1,7 \mathrm{~L}$, esta se prefiltró a través de filtros de $40 \mathrm{~mm}$ de diámetro con un poro de $1 \mathrm{~mm}$ de tamaño para eliminar cualquier contaminante; posteriormente se 
utilizó un filtro Millipore Sterivex de 0,22 $\mu \mathrm{m}$ para realizar la concentración de los microorganismos presentes en la muestra. Finalmente, los filtros se transfirieron a tubos de plástico estériles que contenían $3 \mathrm{~mL}$ del reactivo de protección Monarch ${ }^{\circledR}$ DNA/RNA Protection Reagent y fueron transportados al laboratorio conservando la cadena de frío para ser almacenados a $-20^{\circ}$ hasta la extracción de ADN.

\section{Protocolo de extracción}

Como método estándar comercial se empleó el DNeasy power water kit (Qia$\mathrm{gen}^{\mathrm{TM}}$ ), siguiendo las recomendaciones del fabricante. El protocolo químico se inició con la extracción de ADN genómico mediante un buffer de extracción preparado con: $1 \%, \mathrm{w} / \mathrm{v}$ bromuro de hexadeciltrimetilamonio (CTAB), $3 \%$, w/v dodecilsulfato sódico (SDS), $100 \mathrm{mM}$ Tris-HCl, $100 \mathrm{mM}$ NaEDTA, $1.5 \mathrm{M} \mathrm{NaCl}, \mathrm{pH}$ 8.0, siguiendo el protocolo de Hassan et al. (2018). Con el objetivo de optimizar las concentraciones y la calidad del material obtenido, se realizaron las siguientes modificaciones: se incrementaron los tiempos de

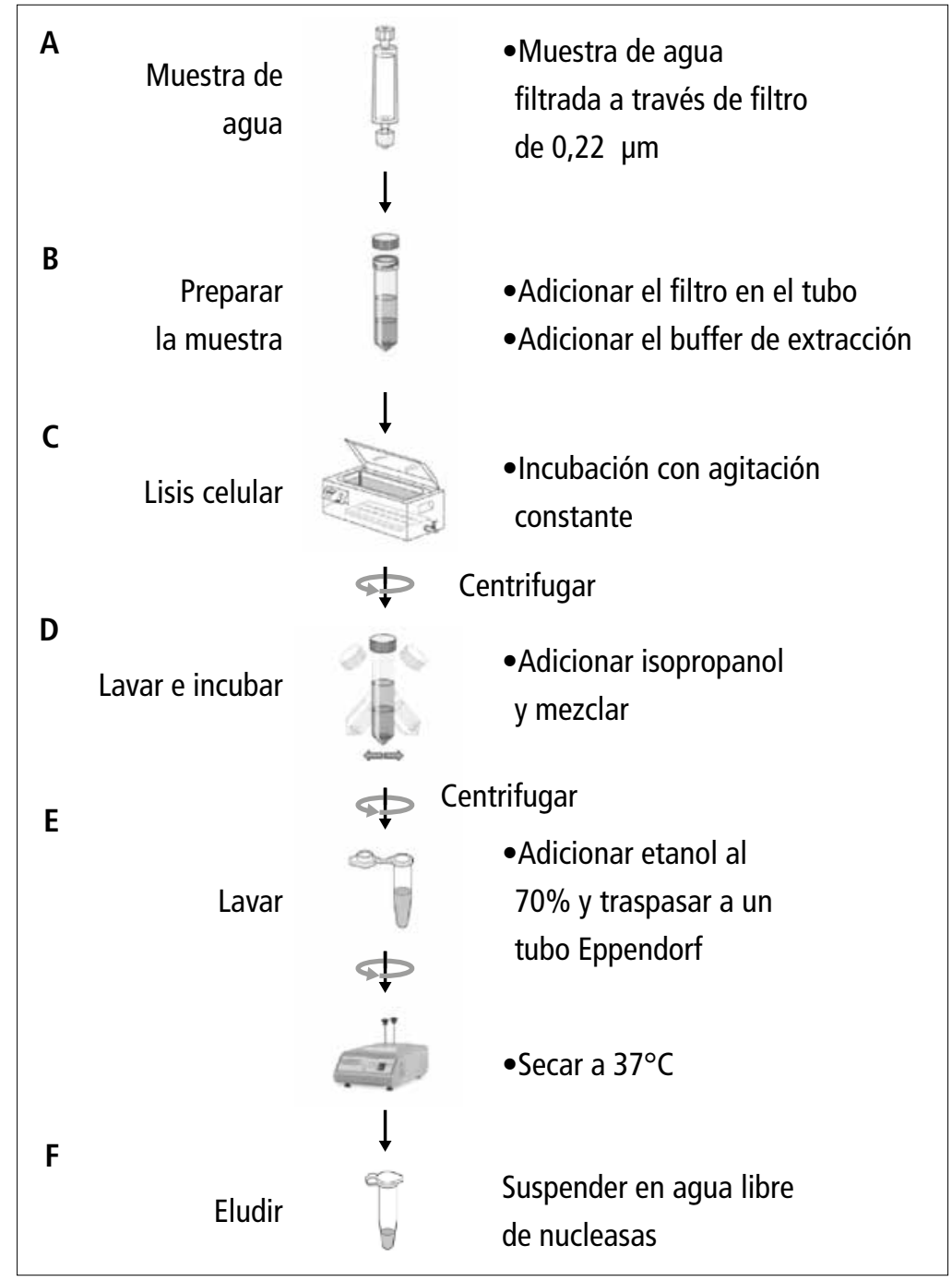

FIGURA 1.

Procedimiento de extracción de ADN a partir de una muestra de agua previamente filtrada.

Fuente: elaboración de los autores. 
centrifugación (15 a $20 \mathrm{~min}$ ) en los pasos (c) y (d), así como el tiempo de incubación a $-4{ }^{\circ} \mathrm{C}(20$ a $25 \mathrm{~min}$ ) en el paso (d). Se adicionaron dos lavados con etanol al $70 \%$ en los pasos (e), y finalmente se resuspendió el ADN obtenido en agua libre de nucleasas (Wizard Cenomic DNA, Promega) (Figura 1).

Los valores de concentración $(\mathrm{ng} / \mathrm{mL}$ ) y calidad (absorbancia 260/280 nm) del ADN genómico extraído se realizó por duplicado en un espectrofotóme-

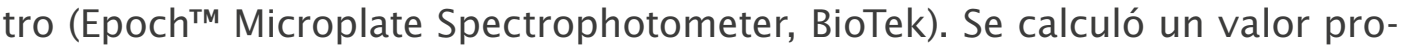
medio de las dos mediciones para cada uno de los parámetros por evaluar, teniendo como objetivo obtener valores superiores a $30 \mathrm{ng} / \mathrm{mL}$ y valores de absorbancia 260/280 superiores a 1,7.

\section{Verificación de ADN bacteriano}

Con el objetivo de validar la calidad del ADN extraído se realizó la amplificación molecular por PCR del fragmento de las regiones hipervariables V3 - V4 (amplicón entre 300 y $459 \mathrm{pb}$ aproximadamente). usando los primeros $341 \mathrm{~F}$ y 785R (Beckers et al., 2016) y el protocolo sugerido en Kiersztyn et al. (2019), con condiciones de termociclado $95^{\circ} \mathrm{C}$ durante 3 minutos, 25 ciclos de $95^{\circ} \mathrm{C}$ durante 30 segundos, $55^{\circ} \mathrm{C}$ durante 30 segundos, $72^{\circ} \mathrm{C}$ durante 30 segundos y un último ciclo de $72^{\circ} \mathrm{C}$ durante 5 minutos. Los resultados se visualizaron en un gel de agarosa al 1,5\%. Se usó como control negativo agua destilada.

\section{Resultados y Discusión}

La relación 260/280 se refiere a la relación de absorbancia de la cantidad de ADN medida a $260 \mathrm{~nm}$ sobre la cantidad de proteína medida a $280 \mathrm{~nm}$. Esta relación es utilizada constantemente para evaluar la pureza del ADN extraído (en la Tabla 1 se muestra la cantidad en $\mathrm{mL}$ de agua de partida que fue filtrada), el grupo de muestra al que corresponde, los valores de concentración (ng/mL) y pureza (relación 260/280) del ADN metagenómico extraído. Entre los parámetros más importantes que se deben tener en cuenta a la hora de observar si un método de extracción es exitoso, se encuentran la pureza, la cantidad y la integridad de las muestras. Al realizar la extracción de ADN con el protocolo químico sin modificar, se observaron concentraciones mayores a $30 \mathrm{ng} / \mathrm{mL}$, pero relaciones de absorbancia $260 / 280$ menores a 1,7; por otro lado, con el kit comercial se obtuvieron concentraciones no mayores a 25.94 $\mathrm{ng} / \mathrm{ml}$, pero con purezas sobre 1,8 . Posterior a esto se hicieron las modificaciones mencionadas anteriormente y se obtuvieron concentraciones mayores a $140 \mathrm{ng} / \mathrm{mL}$, y con una relación de absorbancia media de $>1,7$; por lo cual, se observa la eficacia de eliminar la contaminación de proteínas del ADN metagenómico junto con altas concentraciones de ADN extraído. Se logró obtener ADN libre de contaminación con ARN, no degradado y con concentración y pureza óptima para poder ser analizado por métodos moleculares. 
TABLA 1. Condiciones de muestra, tipo de extracción y valores de concentración y pureza de ADN genómico extraído

\begin{tabular}{|c|c|c|c|c|}
\hline Fuente hídrica & $\begin{array}{c}\text { Volumen filtrado } \\
(\mathbf{m L})\end{array}$ & $\begin{array}{c}\text { Modificación del protocolo } \\
\text { de extracción }\end{array}$ & $\begin{array}{c}\text { Concentración de ADN } \\
\text { (ng/mL) }\end{array}$ & $\begin{array}{c}\text { Pureza de ADN } \\
(\mathbf{2 6 0 / 2 8 0})\end{array}$ \\
\hline Total & 1450 & Original & 45,441 & 1,045 \\
\hline Tota & 1400 & Primera modificación & 91,475 & 1,644 \\
\hline Tota & 1500 & Segunda modificación & 277,293 & $>1,7$ \\
\hline
\end{tabular}

La verificación de ADN bacteriano obtenido se muestra en la Figura 2. Donde se observó la presencia de un amplificado de aproximadamente 320 pb. La ausencia de ADN degradado en la muestra indica la integridad del material extraído, además se aprecia una buena calidad y pureza que permiten realizar su análisis por técnicas moleculares basadas en amplificaciones por PCR.

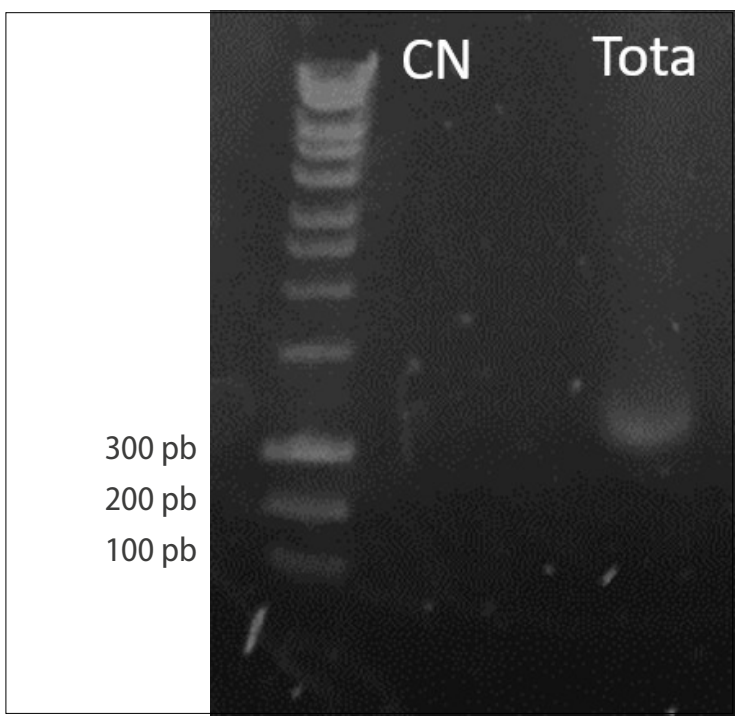

FIGURA 2. Electroforesis en gel de agarosa al $1 \%$ con amplificación de la región V3-V4 el gen rRNA en muestras de ADN. Los carriles muestras: 1. Marcador de peso, 2. Control negativo, 3. Muestra tomada de TOTA y extraída por el protocolo químico usado.

La constante actualización de los métodos moleculares y de análisis genético conlleva la necesidad de desarrollar métodos de extracción de ADN que sean simples, eficientes y de bajo costo. Este aspecto es especialmente importante en los procesamientos de muestras ambientales como suelos, fluidos de desecho y cuerpos de agua, puesto que la complejidad de componentes químicos puede afectar la eficiencia de la lisis celular y la pureza e integridad del ADN obtenido, lo cual a su vez influye radicalmente en los resultados en procedimientos subsiguientes como la PCR y la secuenciación, entre otros.

Debido a las caracteristicas del tipo de muestra de partida (i. e., alto contenido de compuestos orgánicos), las modificaciones presentadas en el protocolo químico hacen referencia al uso del bromuro de cetiltrimetilamonio (CTAB) y el dodecilsulfato sódico (SDS) para la depuración de contaminantes fenólicos 
y sustancias orgánicas de otros protocolos de extracción (Bag et al., 2016; Miao et al., 2014), y al incremento de tiempos de centrifugación y lavados finales. La modificación de este protocolo evitó la utilizacion de disolventes orgánicos altamente contaminantes y difíciles de eliminar como el fenol-cloroformo, el cual es ampliamente usado en protocolos de extraccion de ADN para estudios metagenómicos (Ranasinghe et al., 2015). También se evitó el uso de tratamientos físicos como el golpeo con perlas, sonicación, presión y maceramiento del filtro en el que se encuentran los organismos, pues se tuvo en cuenta que se ha demostrado que estos tratamientos pueden causar daños al ADN obtenido y podría afectarlo para un procesamiento posterior (Sagar et al., 2014).

Nuestros resultados sugieren que este protocolo de extracción de ADN es un método rápido para la obtención de ADN de alta calidad y pureza, alternativo al uso de kits comerciales, y el cual puede ser utilizado en muestras de cuerpos de agua con alto contenido orgánico. Este protocolo puede ser usado antes de cualquier técnica molecular que se emplee como herramienta de biomonitoreo de calidad del agua (Pawlowski et al., 2018), efectos en la salud y productividad de cultivos (Schmidt et al., 2019), así como para otros estudios que pretendan el conocimiento de los patrones de diversidad de microorganismos asociados a características fisicoquímicas de los cultivos y a los ciclos biogeoquímicos (Causarano et al., 2007; Chaffron et al., 2010).

\section{Conclusión}

El protocolo presentado en este trabajo ofrece una opción sencilla y de bajo costo para la extracción de ADN de muestras ambientales de agua. También vale señalar que modificar y ajustar los protocolos existentes de acuerdo con las necesidades y características propias de las muestras, pueden llegar a aumentar su rendimiento en términos de concentración y pureza del ADN extraído.

\section{AGRADECIMIENTOS}

Los autores expresan su agradecimiento a la Dirección de Investigaciones de la Universidad Pedagógica y Tecnológica de Colombia en el marco de la Convocatoria DIN número 8 de 2019, y al grupo de investigación UDESA, por el apoyo logístico en la toma de muestras y en la realización de esta investigación. 


\section{Referencias}

Bag, S., Saha, B., Mehta, O., Anbumani, D., Kumar, N., Dayal, M., .. . Das, B. (2016). An Improved Method for High Quality Metagenomics DNA Extraction from Human and Environmental Samples. Scientific Reports, 6, 26775-26775. http:// dx.doi.org/10.1038/srep26775

Beckers, B., Op De Beeck, M., Thijs, S., Truyens, S., Weyens, N., Boerjan, W., \& Vangronsveld, J. (2016). Performance of 16s rDNA Primer Pairs in the Study of Rhizosphere and Endosphere Bacterial Microbiomes in Metabarcoding Studies. Frontiers in Microbiology, 7, 650-650. http://dx.doi.org/10.3389/fmicb.2016.00650

Bohorquez, L. C., Delgado-Serrano, L., López, G., Osorio-Forero, C., Klepac-Ceraj, V., Kolter, R., . . . Zambrano, M. M. (2012). In-depth Characterization via Complementing Culture-Independent Approaches of the Microbial Community in an Acidic Hot Spring of the Colombian Andes. Microbial Ecology, 63(1), 103-11 1. http://dx.doi.org/10.1007/s00248-011-9943-3

Boon, E., Whidden, C., Langille, M. G. I., Beiko, R. G., Meehan, C. J., \& Wong, D. H. J. (2014). Interactions in the Microbiome: Communities of Organisms and Communities of Genes. FEMS Microbiology Reviews, 38(1), 90-118. http://dx.doi. org/10.1111/1574-6976.12035

Causarano, H. J., Shaw, J. N., Franzluebbers, A. J., Reeves, D. W., Raper, R. L., Balkcom, K. S., . . . Izaurralde, R. C. (2007). Simulating Field-Scale Soil Organic Carbon Dynamics Using EPIC. Soil Science Societies of America Journal, 71(4), 1174-1 185. http://dx.doi.org/10.2136/sssaj2006.0356

Chaffron, S., Rehrauer, H., Pernthaler, J., \& Von Mering, C. J. G. r. (2010). A Global Network of Coexisting Microbes from Environmental and Whole-Genome Sequence Data. Genome Res., 20(7), 947-959.

Chaudhary, A., Kauser, I., Ray, A., \& Poretsky, R. (2018). Taxon-Driven Functional Shifts Associated with Storm Flow in an Urban Stream Microbial Community. mSphere, 3(4), e00194-00118. http://dx.doi.org/10.1128/mSphere.00194-18

Delgado-Serrano, L., López, G., Bohorquez, L. C., Bustos, J. R., Rubiano, C., Osorio-Forero, C., . . Zambrano, M. M. (2014). Neotropical Andes Hot Springs Harbor Diverse and Distinct Planktonic Microbial Communities. FEMS Microbiology Ecology, 89(1), 56-66. http://dx.doi.org/10.1111/1574-6941.12333 \%J FEMS Microbiology Ecology

Fennell, D. E., Du, S., Liu, F., Liu, H., \& Häggblom, M. M. (2011). Dehalogenation of Polychlorinated Dibenzo-p-Dioxins and Dibenzofurans, Polychlorinated Biphenyls, and Brominated Flame Retardants, and Potential as a Bioremediation Strategy. Pergamon Press.

Fuhrman, J. A. (2009). Microbial Community Structure and its Functional Implications. Nature, 459(7244), 193-199. http://dx.doi.org/10.1038/nature08058

George, I., Stenuit, B., Agathos, S., \& Marco, D. (2010). Application of Metagenomics to Bioremediation. Metagenomics: Theory, Methods and Applications, 1, 119-140.

Gilbert, J. A., Jansson, J. K., \& Knight, R. (2014). The Earth Microbiome Project: Successes and Aspirations. BMC Biology, 12(1), 69. http://dx.doi.org/10.1186/ s12915-014-0069-1 
Gilbert, J. A., Meyer, F., Field, D., Schriml, L. M., \& Garrity, G. M. (2010). Metagenomics: A Foundling Finds Its Feet. Standards in Genomic Sciences, 3(2), 212-213. http://dx.doi.org/10.4056/sigs. 1213842

Hassan, M., Essam, T., \& Megahed, S. (2018). Illumina Sequencing and Assessment of New Cost-Efficient Protocol for Metagenomic-DNA Extraction from Environmental Water Samples. Brazilian Journal of Microbiology: [publication of the Brazilian Society for Microbiology], 49 Suppl 1(Suppl 1), 1-8. http://dx.doi. org/10.1016/j.bjm.2018.03.002

Hu, Y., Liu, Z., Yan, J., Qi, X., Li, J., Zhong, S., . . . Liu, Q. (2010). A Developed DNA Extraction Method for Different Soil Samples. Journal of Basic Microbiology, 50(4), $401-407$.

Huang, Q., Briggs, B. R., Dong, H., Jiang, H., Wu, G., Edwardson, C., . . . Quake, S. (2014). Taxonomic and Functional Diversity Provides Insight into Microbial Pathways and Stress Responses in the Saline Qinghai Lake, China. PLoS ONE, 9(11), 1-10. http://dx.doi.org/10.1371/journal.pone.0111681

Jiménez, D. J., Andreote, F. D., Chaves, D., Montaña, J. S., Osorio-Forero, C., Junca, H., ... Baena, S. (2012). Structural and Functional Insights from the Metagenome of an Acidic Hot Spring Microbial Planktonic Community in the Colombian Andes. PLoS ONE, 7(12), 1-15. http://dx.doi.org/10.1371/journal.pone.0052069

Jiménez, D. J., Montaña, J. S., Álvarez, D., \& Baena, S. (2012). A Novel Cold Active Esterase Derived from Colombian High Andean Forest Soil Metagenome. World Journal of Microbiology and Biotechnology, 28(1), 361-370. http://dx.doi. org/10.1007/s $11274-011-0828-x$

Kathiravan, M. N., Gim, G. H., Ryu, J., Kim, P. I., Lee, C. W., \& Kim, S. W. (2015). Enhanced Method for Microbial Community DNA Extraction and Purification from Agricultural Yellow Loess Soil. J Microbiol, 53(1 1), 767-775. http://dx.doi. org/10.1007/s12275-015-5454-0

Kiersztyn, B., Chróst, R., Kaliński, T., Siuda, W., Bukowska, A., Kowalczyk, G., \& Grabowska, K. (2019). Structural and Functional Microbial Diversity Along A Eutrophication Gradient of Interconnected Lakes Undergoing Anthropopressure. Scientific Reports, 9(1), 11144-11144. http://dx.doi.org/10.1038/ s41598-019-47577-8

Miao, T., Gao, S., Jiang, S., Kan, G., Liu, P., Wu, X., . . Yao, S. (2014). A Method Suitable for DNA Extraction from Humus-Rich Soil. Biotechnology Letters, 36(11), 2223-2228.

Mouillot, D., Graham, N. A. J., Villéger, S., Mason, N. W. H., \& Bellwood, D. R. (2013). A functional Approach Reveals Community Responses to Disturbances. Trends In Ecology \& Evolution, 28(3), 167-177.

Pawlowski, J., Kelly-Quinn, M., Altermatt, F., Apothéloz-Perret-Gentil, L., Beja, P., Boggero, A., ... Kahlert, M. (2018). The Futureof Biotic Indices in the Ecogenomic Era: Integrating (e)DNA Metabarcoding in Biological Assessment of Aquatic Ecosystems. Science of the Total Environment, 637-638, 1295-1310. http://dx. doi.org/10.1016/j.scitotenv.2018.05.002 
Payne, J. T., Millar, J. J., Jackson, C. R., \& Ochs, C. A. (2017). Patterns of Variation in Diversity of the Mississippi River Microbiome over 1,300 Kilometers. PloS One, 12(3), e0174890-e0174890. http://dx.doi.org/10.1371/journal.pone.0174890

Peter, H., \& Sommaruga, R. (2016). Shifts in Diversity and Function of Lake Bacterial Communities upon Glacier Retreat. The ISME Journal, 10(7), 1545-1554. http:// dx.doi.org/10.1038/ismej.2015.245

Ranasinghe, C. P., Harding, R., \& Hargreaves, M. (2015). An Improved Protocol for the Isolation of Total Genomic DNA from Labyrinthulomycetes. Biotechnology Letters, 37(3), 685-690.

Reed, H. E., \& Martiny, J. B. H. (2013). Microbial Composition Affects the Functioning of Estuarine Sediments. The ISME Journal, 7(4), 868-868.

Röling, W. F. M., Van Breukelen, B. M., Bruggeman, F. J., \& Westerhoff, H. V. (2007). Ecological Control Analysis: Being (S) in Control of Mass Flux and Metabolite Concentrations in Anaerobic Degradation Processes. Environmental Microbiology, 9(2), 500-511.

Sagar, K., Singh, S. P., Goutam, K. K., \& Konwar, B. K. (2014). Assessment of Five Soil DNA Extraction Methods and a Rapid Laboratory-Developed Method for Quality Soil DNA Extraction for 165 rDNA-Based Amplification and Library Construction. Journal of Microbiological Methods, 97, 68-73.

Schmidt, J. E., Kent, A. D., Brisson, V. L., \& Gaudin, A. C. M. (2019). Agricultural Management and Plant Selection Interactively Affect Rhizosphere Microbial Community Structure and Nitrogen Cycling. Microbiome, 7(1), 146. http://dx. doi.org/10.1186/s40168-019-0756-9

Venter, J. C., Remington, K., Heidelberg, J. F., Halpern, A. L., Rusch, D., Eisen, J. A., . . . Nelson, W. (2004). Environmental Genome Shotgun Sequencing of the Sargasso Sea. Science, 304(5667), 66-74.

Zelaya-Molina, L. X., Ortega, M. A., \& Dorrance, A. E. (2011). Easy and Efficient Protocol for Oomycete DNA Extraction Suitable for Population Genetic Analysis. Biotechnology Letters, 33(4), 715-720. 